\title{
NOTE ON THE PROPAGATION OF DISTURBANCES IN A LIQUID CONTAINING GAS BUBBLES
}

\author{
by J. D. MURRAY \\ Eng. mechs. Dept., University of Michigan, Ann Arbor, Michigan, U.S.A.
}

\section{Summary}

In a liquid containing gas bubbles the speed of sound is less than that in each phase separately. Using the equations of motion for a homogeneous liquid containing gas bubbles it is shown that the dominating attenuation. of an infinitesimal disturbance is that due to the second viscosity. In the propagation of a finite compressive disturbance an expression for the time required for the disturbance to display shock characteristics is found in terms of the initial disturbance profile and the liquid-gas ratio.

§ 1. Introduction. Mallock $\mathrm{k}^{1}$ ) evaluated the speed of sound in a liquid containing gas bubbles assuming Boyle's law held for the gas. For a large range of conditions he showed that the speed of sound was less than that of the liquid or the gas separately. Hsieh and Plesset ${ }^{2}$ ) repeated the evaluation of the speed of sound but restricted themselves to the region where the ratio of the masses of gas to liquid was small, and they found the attenuation of an infinitesimal wave, due to heat conduction. Campbell and Pitcher ${ }^{3}$ ) considered theoretically and experimentally the motion and collision of fully developed shock waves in such a mixture.

This note uses the equations of motion of the mixture to obtain for reference Mallock's results except that, in the following, the adiabatic, rather than the isothermal, speed of sound in the gas is the limiting case. The attenuation of an infinitesimal disturbance due to the bulk or second viscosity of the liquid-gas mixture is evaluated and it is shown that, unlike the pure gas case, the attenuation due to heat conduction is negligible in comparison. The 
propagation of finite amplitude waves is considered and an expression is found for the time required for a compressive wave to develop shock wave features, as a function of the liquid-gas ratio and the initial disturbance profile.

$\S 2$. Equations of motion. The mixture of most interest is that of liquid-gas components consisting of a liquid, in which gas bubbles are uniformly distributed which are small enough so that the resulting mixture may be considered homogeneous and isotropic. The bubble size must be small compared with any disturbance. This condition implies that the mixture acquires the property of a foam as the proportion of gas increases.

Denote the liquid and gas components by suffixes 1 and 2 respectively, and the volumes, masses and densities by $V_{1}, V_{2}, M_{1}$, $M_{2}$ and $\rho_{1}, \rho_{2}$. Introduce

$$
\xi=V_{2} / V_{1}, \quad \eta=M_{2} / M_{1}, \quad \rho_{2} / \rho_{1}=\eta / \xi=\text { a constant. }
$$

The homogeneous mixture density, $\rho$, is given by

$$
\begin{aligned}
\frac{1}{\rho} & =\frac{V_{1}+V_{2}}{M_{1}+M_{2}}= \\
& =\frac{1}{\rho_{1}}\left(\frac{1+\xi}{1+\eta}\right)=\frac{1}{\rho_{2}}\left(\frac{1+\xi}{1+\eta}\right) \frac{\eta}{\xi}=\frac{1}{(1+\eta)}\left(\frac{1}{\rho_{1}}+\frac{\eta}{\rho_{2}}\right),
\end{aligned}
$$

the coefficient of heat conduction, $K$, by

$$
K=\frac{K_{1} V_{1}+K_{2} V_{2}}{V_{1}+V_{2}}=\frac{K_{1}+\xi K_{2}}{1+\xi},
$$

the specific heat, $c$, by

$$
c=\frac{1}{\rho}\left(\frac{M_{1} c_{1}+M_{2} c_{2}}{V_{1}+V_{2}}\right)=\frac{c_{1}+\eta c_{2}}{1+\eta},
$$

where $c_{2}$ is the specific heat at constant volume. The second vis$\left.\operatorname{cosity}^{4}\right) \mu^{\prime}$ is particularly important in liquid-gas mixtures. Ta ylor ${ }^{5}$ ) and Davies ${ }^{6}$ ) have shown that

$$
\mu^{\prime}=\frac{4}{3} \frac{\mu_{1}(1+\xi) \xi y^{2}}{(1+\xi+\xi y)^{2}}
$$

where $\mu_{1}$ is the shear viscosity of the liquid, and $y$ the ratio of the 
effective compressibility of the gas to that of the liquid. The equations of motion for the mixture are

$$
\begin{gathered}
\frac{\partial \rho}{\partial t}+\boldsymbol{\nabla} \cdot \rho \boldsymbol{v}=0, \\
\rho \frac{\mathrm{d} \boldsymbol{v}}{\mathrm{d} t}=-\boldsymbol{\nabla} p-\boldsymbol{\nabla} \times\left(\boldsymbol{\nabla} \times \mu_{1} \boldsymbol{v}\right)+\boldsymbol{\nabla}\left[\left(\mu^{\prime}\right) \boldsymbol{\nabla} \cdot \boldsymbol{v}\right], \\
\rho c \frac{\mathrm{d} T}{\mathrm{~d} t}+p \boldsymbol{\nabla} \cdot \boldsymbol{v}=K \boldsymbol{V}^{2} T+\mu_{1}\left[\boldsymbol{\nabla} \cdot\left(\boldsymbol{\nabla} \boldsymbol{v}^{2}+2 \boldsymbol{\omega} \times \boldsymbol{v}\right)-\right. \\
\left.-2(\boldsymbol{v} \cdot \boldsymbol{\nabla})(\boldsymbol{\nabla} \cdot \boldsymbol{v})+\boldsymbol{\omega}^{2}\right]+\mu^{\prime}(\boldsymbol{\nabla} \cdot \boldsymbol{v})^{2}, \\
p=F_{1}\left(\rho_{1}, T\right), \\
p=R_{2 \rho_{2}} T,
\end{gathered}
$$

where $\boldsymbol{v}$ is the velocity, $\omega$ the vorticity, $p$ the pressure, $T$ the temperature, $R_{2}$ the gas constant, $F_{1}$ the equation of state for the liquid and $\rho, K, c$ and $\mu^{\prime}$ given by equations (2)-(5). The omission of $\mu_{1}$, in comparison with $\mu^{\prime}$ in the last term of (7) is consistent with the derivation of $\mu^{\prime}$, given by (5).

§3. Speed of sound. In the usual way, the equations are linearised on putting

$$
\begin{array}{ll}
\rho_{1}=\rho_{01}\left(1+s_{1}\right), & \rho_{2}=\rho_{02}\left(1+s_{2}\right), \quad \rho=\rho_{0}(1+s), \\
T=T_{0}(1+\tau), \quad p=p_{0}(1+\tau), &
\end{array}
$$

where $\rho_{01}, \rho_{02}, \rho_{0}, T_{0}, p_{0}$ are the rest values and $s_{1}, s_{2}, s, \tau, \pi, v$ (and $\boldsymbol{\omega}$ ) are small. Second order terms are neglected. Equation (10) gives

and (9) gives

$$
\pi=s_{2}+\tau,
$$

where

$$
\pi=a_{1} s_{1}+b_{1} \tau
$$

$\left.\left.a_{1}=\frac{\rho_{01}}{p_{0}} \frac{\partial F_{1}\left(\rho_{1}, T\right)}{\partial \rho_{1}}\right]_{\substack{T=T_{0} \\ \rho_{1}=\rho_{01}}}, \quad b_{1}=\frac{T_{0}}{p_{0}} \frac{\partial F_{1}\left(\rho_{1}, T\right)}{\partial T}\right]_{\substack{T=T_{0}=\rho_{01} \\ \rho_{1}}}$.

Equation (2) becomes

$$
\frac{s}{\rho_{0}}=\frac{1}{\rho_{02}} \frac{\eta}{(1+\eta)}\left(\frac{s_{1}+\xi s_{2}}{\xi}\right)=\frac{1}{\rho_{01}}\left(\frac{s_{1}+\xi s_{2}}{1+\eta}\right) .
$$


Equations (12), (13) and (15) now give

$$
\pi=a s+b \tau \text {, }
$$

where

$$
a=\frac{a_{1}(1+\xi)}{\left(1+a_{1} \xi\right)}, \quad b=\frac{b_{1}+a_{1} \xi}{1+a_{1} \xi} .
$$

The governing equations are now (16), (18), (19) and (20), the last three being the linearised form of (6), (7) and (8), namely,

$$
\begin{gathered}
\frac{\partial s}{\partial t}+\boldsymbol{\nabla} \cdot \boldsymbol{v}=0 \\
\frac{\partial \boldsymbol{v}}{\partial t}+\frac{p_{0}}{\rho_{0}} \boldsymbol{\nabla} \pi+\boldsymbol{X}=0, \\
\nabla^{2} \tau-\frac{1}{d} \frac{\partial \tau}{\partial t}-\frac{p_{0}}{K T_{0}} \boldsymbol{\nabla} \cdot \boldsymbol{v}=0,
\end{gathered}
$$

where $d$, the effective thermal conductivity is given by

$$
d=\frac{K}{\rho_{0} c},
$$

and $\boldsymbol{X}$, the viscous term by

$$
\boldsymbol{X}=-\frac{1}{\rho_{0}}\left[\mu_{1} \nabla^{2} \boldsymbol{v}+\mu^{\prime} \boldsymbol{\nabla}(\boldsymbol{\nabla} \cdot \boldsymbol{v})\right] .
$$

From (16), (17), (18), (19), the $v$-equation is

$$
\begin{aligned}
\frac{1}{d} \frac{\partial}{\partial t}\left[\frac{\partial^{2} \boldsymbol{v}}{\partial t^{2}}+\frac{\partial \boldsymbol{X}}{\partial t}-\right. & \left.\frac{p_{0} a}{\rho_{0}}(1+\alpha) \boldsymbol{\nabla}(\boldsymbol{\nabla} \cdot \boldsymbol{v})\right], \\
& =\nabla^{2}\left[\frac{\partial^{2} \boldsymbol{v}}{\partial t^{2}}+\frac{\partial \boldsymbol{X}}{\partial t}-\frac{p_{0} a}{\rho_{0}} \boldsymbol{\nabla}(\boldsymbol{\nabla} \cdot \boldsymbol{v})\right],
\end{aligned}
$$

where

$$
\alpha=\frac{p_{0} b d}{a K T_{0}}=\frac{\left(b_{1} / a_{1}+\xi\right)\left(\gamma_{2}-1\right) \eta}{\left(\eta+c_{1} / c_{2}\right) \xi}=\frac{\left(b_{1} / a_{1}+\xi\right) p_{0}}{\left(1+c_{2} \eta / c_{1}\right) \rho_{01} c_{1} T_{0}},
$$

where $\gamma_{2}$ is the ratio of specific heats of the gas. If the liquid acts as a constant temperature reservoir, (9) may be taken as

$$
p-p_{0}=l\left(\rho-\rho_{0}\right) / \rho_{0}
$$


which, from (13), gives

$$
\pi=\frac{l}{p_{0}} s_{1}
$$

where $l$ is the elasticity of volume. In the above expression for $\alpha$, $b_{1} / a_{1}$ is zero*) and

$$
\alpha=\frac{\eta\left(\gamma_{2}-1\right)}{\eta+c_{1} / c_{2}}=\frac{\xi}{c_{1}+c_{2} \eta}\left(-\frac{p_{0}}{\rho_{01} T_{0}}\right) .
$$

If we neglect the viscous term for the present (it is discussed in $\S 4)$ then the isothermal limit gives the right hand side of (23) zero: that is

$$
\frac{\partial^{2} \boldsymbol{v}}{\partial t^{2}}-\frac{p a}{\rho_{0}} \boldsymbol{\nabla}(\boldsymbol{V} \cdot \boldsymbol{v})=0 .
$$

The isothermal limit occurs when $|(1 / d) \partial \tau / \partial t| \ll\left|\left(p_{0} / K T_{0}\right) \boldsymbol{\nabla} \cdot \boldsymbol{v}\right|$ in (20). From (25) the isothermal speed of sound $c_{i}$ is given by

$$
c_{i}^{2}=p a / \rho_{0} .
$$

The adiabatic limit is obtained on putting $\left|\nabla^{2} \tau\right| \ll\left|\left(p_{0} / K T_{0}\right) \boldsymbol{\nabla} \cdot \boldsymbol{v}\right|$, which gives the left hand side of (23) zero, namely

$$
\frac{\partial^{2} \boldsymbol{v}}{\partial t^{2}}-\frac{p a}{\rho_{0}}(1+\alpha) \boldsymbol{\nabla}(\boldsymbol{\nabla} \cdot \boldsymbol{v})=0,
$$

from which the adiabatic speed of sound $c_{a}$ is given by

$$
c_{a}^{2}=\frac{p a}{\rho_{0}}(1+\alpha) .
$$

Over a large range of interest $\eta \leqslant 1$ and $c_{1} / c_{2}$ is $O(1)$ and from (24) $\alpha \ll 1$. Thus in the range where the mixture is distinctly that of a liquid with a distribution of bubbles (rather than a foam)

$$
c_{a} \fallingdotseq c_{i}
$$

and the speed of sound is effectively the isothermal speed of sound. However, if $\eta \rightarrow \infty$, (27), (24) and (17) give $c_{a}=\gamma_{2} p / \rho_{02}$ which is the adiabatic speed of sound in the gas. In fact, as we see below in $\S 4$ the speed of sound is strictly the adiabatic speed of sound, but as shown above over a wide range this differs negligibly from

*) In most liquids over a wide range of interest if $b_{1} \not \equiv 0, b_{1} \ll a_{1}$. 
the isothermal speed of sound. Fig. 1 shows $c_{\alpha}$ as a function of $\xi$, the units for $c_{a}$ being $\mathrm{cm} / \mathrm{sec}$.

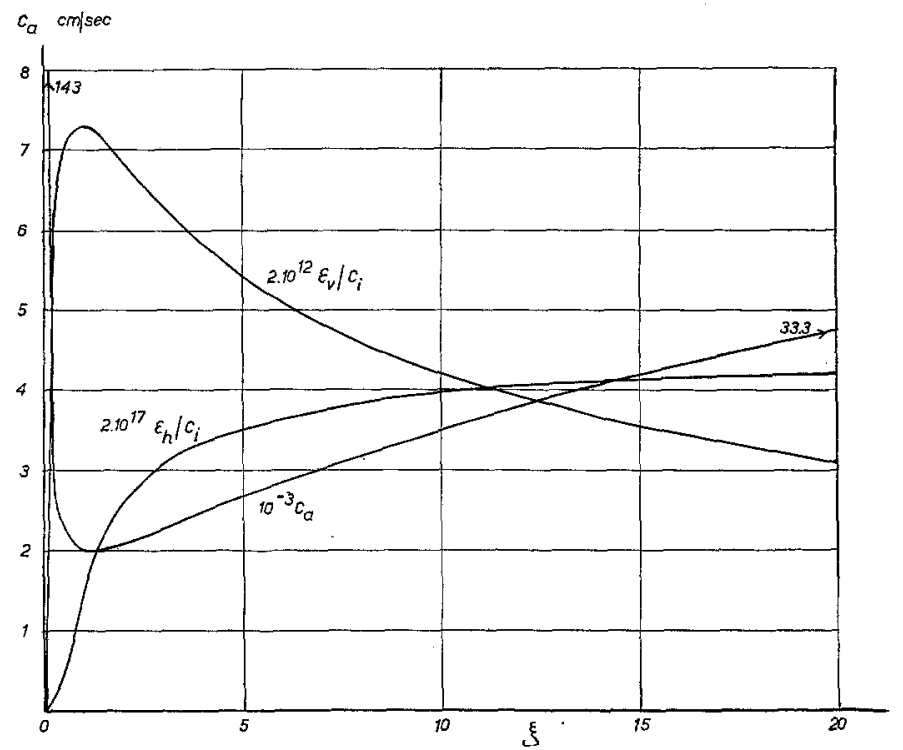

Fig. 1. Adiabatic velocity of sound and attenuation factors versus gas/liquid volume ratio.

That $c_{a}$ in (27) is less than the speed of sound in either the liquid or gas alone is easily shown, since $a_{1} \geqslant 1$ and for $\xi$ of $O(1)$, say,

$$
\begin{aligned}
\frac{p_{0} a(1+\alpha)}{\rho_{0}} & =\left(\frac{p_{0} a_{1}}{\rho_{01}}\right) \frac{(1+\xi)^{2}}{\left(1+a_{1} \xi\right)(1+\eta)}\left[1+\frac{\eta\left(\gamma_{2}-1\right)}{\eta+c_{1} / c_{2}}\right] \fallingdotseq \\
& \fallingdotseq\left(\frac{p_{0} a_{1}}{\rho_{01}}\right) \frac{1}{a_{1} \xi} \frac{(1+\xi)^{2}}{(1+\eta)}\left[\frac{\gamma_{2} \eta+c_{1} / c_{2}}{\eta+c_{1} / c_{2}}\right] \ll\left(\frac{p_{0} a_{1}}{\rho_{01}}\right)= \\
& =\text { (speed of sound in the liquid })^{2} .
\end{aligned}
$$

On the other hand, for $\xi \gg 1, \eta \gg 1$, we have $\eta / \xi=\rho_{02} / \rho_{01} \ll 1$, and

$$
\begin{aligned}
\frac{p_{0} a(1+\alpha)}{\rho_{0}} & =\frac{p_{0}}{\rho_{02}} \frac{a_{1}(1+\xi)^{2}}{\left(1+a_{1} \xi\right)(1+\eta)} \frac{\eta}{\xi}\left[\frac{\gamma_{2} \eta+c_{1} / c_{2}}{\eta+c_{1} / c_{2}}\right] \fallingdotseq \\
& \fallingdotseq\left(\frac{\gamma_{2} p_{0}}{\rho_{02}}\right) \frac{(1+1 / \xi)^{2}}{(1+1 / \eta)}\left[\frac{1+c_{1} / \gamma_{2} c_{2} \eta}{1+c_{1} / c_{2} \eta}\right] \fallingdotseq \\
& \fallingdotseq\left(\frac{\gamma_{2} p_{0}}{\rho_{02}}\right)\left[1+\left(\frac{2}{\xi}-\frac{1}{\eta}\right)+\frac{c_{1}\left(1-\gamma_{2}\right)}{c_{2} \gamma_{2} \eta}\right]< \\
& <\text { (speed of sound in the gas) })^{2}
\end{aligned}
$$


since $(2 / \xi-1 / \eta)=(2 \eta / \xi-1) .1 / \eta<0$, and $\left(1-\gamma_{2}\right)<0$. As an example, in a water-air mixture at N.T.P. with $\xi=0.2, \rho_{0} \fallingdotseq$ $\fallingdotseq 0.834 \mathrm{~g} / \mathrm{cm}^{3}, a \fallingdotseq 5$ and $c_{i} \fallingdotseq 2.5 \times 10^{3} \mathrm{~cm} / \mathrm{s}, \quad c_{\text {water }} \fallingdotseq 1.4 \times$ $\times 10^{5} \mathrm{~cm} / \mathrm{s}, c_{\text {air }} \fallingdotseq 3.3 \times 10^{4} \mathrm{~cm} / \mathrm{s}$.

$\S 4$. Attenuation of sound waves. Consider an advancing plane wave in the positive $x$-direction given by

$$
v=\exp i(k x-\omega t)=\exp i k(x-c t), \quad c=\omega / k .
$$

The $x$-component of (23) gives

$$
\frac{i \omega}{d}\left[\omega^{2}+i \nu k^{2}-c_{i}^{2}(1+\alpha) k^{2}\right]=k^{2}\left[\omega^{2}+i \nu k^{2} \omega-c_{i}^{2} k^{2}\right],
$$

where $\nu=\mu^{\prime} / \rho_{0}$ and the $\mu_{1}$ contribution in (22) has been neglected compared with the $\mu^{\prime}$ contribution, consistent with the derivation of $\left.(5)^{5}\right)$. Since $c=\omega / k$ (the speed of the wave), the last equation becomes

$$
c^{3}+i k(v+d) c^{2}-\left[c_{i}^{2}(1+\alpha)+k^{2} v d\right] c-i k d c_{i}^{2}=0 .
$$

Since $v, d, v d$ are, to a first approximation, negligible compared with $c_{i}^{2}$, (29) gives

$$
c^{2}=c_{i}^{2}(1+\alpha) \doteqdot c_{i}^{2}
$$

over a wide range (since $\alpha \leqslant 1$ ). Thus, for $\alpha$ not negligible, the speed of sound in the mixture is the abiabatic speed of sound. In cases of interest, however, $\alpha \ll 1$.

In the pure gas case the second order effects due to the viscosity and heat conduction are comparable. To consider these effects, put

$$
k^{2}=\left(\frac{\omega}{c_{i}}\right)^{2}(1+\varepsilon)^{2},
$$

where $\varepsilon$ is a small correction. Equation (29) gives

$$
\begin{aligned}
\varepsilon & =-\frac{\alpha}{1+\left(\frac{\omega d}{c_{i}}\right)^{2}}+i \frac{1}{2} \frac{\omega}{c_{i}^{2}}\left[\frac{\alpha d}{1+\left(\frac{\omega d}{c_{i}}\right)^{2}}+\frac{\nu}{1+\left(\frac{\omega d}{c_{i}}\right)^{2}}\right], \\
& =\varepsilon_{1}+i \omega\left(\varepsilon_{h}+\varepsilon_{\nu}\right),
\end{aligned}
$$

where $\omega \varepsilon_{1} / c_{i}, \omega^{2} \varepsilon_{h} / c_{i}, \omega^{2} \varepsilon_{w} / c_{i}$ are defined by (31) and represent respectively, the correction to the velocity of propagation due to $\alpha^{*}$ ),

*) It appears as a correction because we assumed in $(30), \alpha \ll 1$. 
the attenuation due to heat conduction and viscosity. Unlike the pure gas case

$$
\varepsilon_{h} \ll \varepsilon_{\nu} .
$$

Fig. 1 gives these attenuation factors for varying $\xi$, and it is seen that the heat conduction correction is a third order effect and in general is less than for the corresponding pure gas case.

As an example, if $\xi=0.2$,

$$
\begin{gathered}
1 / \rho_{0} \doteqdot 1.2 \mathrm{~cm}^{3} / \mathrm{g}, \quad \eta \div 2.5 \times 10^{-4}, \quad c_{i} \doteqdot 2.5 \times 10^{3} \mathrm{~cm} / \mathrm{sec} \\
\alpha \doteqdot 1.75 \times 10^{-5}, \quad d \doteqdot\left(K_{1}+\xi K_{2}\right) / c_{1} \doteqdot 1.42 \times 10^{-3} \mathrm{~cm}^{2} / \mathrm{sec} \\
\mu^{\prime} \doteqdot 1.43 \times 10^{-1} \text { dyne } / \mathrm{cm}^{2} / \text { unit velocity grad }
\end{gathered}
$$

and the attenuation factors for a wave of the form in (28) are from (30) and (31),

$$
\frac{\omega^{2}}{c_{i}} \varepsilon_{h} \doteqdot \omega^{2} 8.2 \times 10^{-19} \mathrm{sec}^{2}, \quad \frac{\omega^{2}}{c_{i}} \varepsilon_{y} \doteqdot \omega^{2} 5.6 \times 10^{-12} \mathrm{sec}^{2},
$$

where $\omega$, the frequency, may vary from $1 / \mathrm{sec}$ to $10^{5} / \mathrm{sec}$.

$\S 5$. Propagation of finite disturbances. The method used is that of Riemann*). The analysis requires a pressure-density law and we formally write for $c$, the speed of sound,

$$
\frac{\mathrm{d} p}{\mathrm{~d} \rho}=c^{2}=\frac{p a(1+\alpha)}{\rho}=\frac{\Gamma p}{\rho},
$$

where $\Gamma=a(1+\alpha)$ with $a$ and $\alpha$ from (17) and (24). From (32) on integrating,

$$
p / \rho^{\Gamma}=\text { constant. }
$$

Following the method of Riemann, if $v=f(x)$ is the wave form at $t=0$, then the advancing wave at $t=t$ is given by

$$
v(x, t)=f\left(x-\left[c_{0}+\frac{1}{2}(\Gamma+1) v\right] t\right),
$$

where in this case $c_{0}=c_{a}$ from (27). Campbell and Pitcher ${ }^{3}$ ) state that a compressive wave should steepen and become a shock wave. From (34) we see that if $f(x)$ is a decreasing function of $x$ then the wave will steepen since, if $v=v_{1}, v_{2}$ are the values at $t=0$ when $x=x_{1}, x_{2}$, the values at $t=t$ are obtained by adding to the $x_{1}, x_{2}$ terms $\left[c_{0}+\frac{1}{2}(\Gamma+1) v_{1}\right] t$ and $\left[c_{0}+\frac{1}{2}(\Gamma+1) v_{2}\right] t$ respectively. The latter is smaller than the former in the compressive case and

*) See, for example, S. Goldstein, Lectures in Fluid Dynamics, Acad. Pres., 1960. 
the wave therefore steepens. A shock wave or discontinuity, first appears when $\partial v / \partial x$ is infinite, after which time the solution (34) would become triply valued over a range, which is physically unrealistic. If $t=t_{s}$ when $\partial v / \partial x$ becomes infinite, differentiating (34) and solving for $t_{s}$ gives

$$
t_{s}=2\left[(\Gamma+1)\left\{1-f^{\prime}(\zeta)_{\max }\right\}\right]^{-1}
$$

where $\zeta=\left[c_{a}+\frac{1}{2}(\Gamma+1) v\right] t$. For $t>t_{s}$ the position of the shock is obtained in the usual way from conservation arguments. In a similar way an expansion wave flattens as it progresses. From (17), (24)

$$
a_{1} \leqslant \Gamma \leqslant \gamma_{2}
$$

and from (35) it is clear that a shock wave appears in a mixture in a shorter time than if the same wave were propagated in the pure gas.

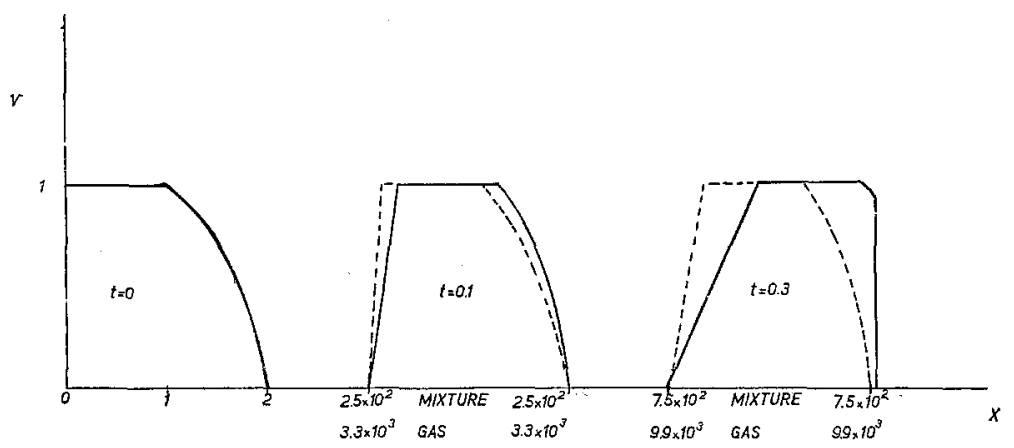

Fig. 2. Wave profile in air (---) and water-air mixture $\xi=2(-)$ at times $t=0,0.1$ and 0.3 .

Campbell and Pitcher ${ }^{3}$ ) show experimentally that an expansive wave of Heaviside form does flatten. Thus to illustrate the effect on a wave, fig. 2 shows the form at various times of a wave initially given by

$$
\begin{aligned}
& v(x, 0)=0 \quad \text { for } x \leqslant 0, \\
& =1 \quad \text { for } 0 \leqslant x \leqslant 1 \text {, } \\
& =\cos \frac{\pi}{2}(x-1) \text { for } 1 \leqslant x \leqslant 2 \text {, } \\
& =0 \quad \text { for } 2 \leqslant x \text {, }
\end{aligned}
$$


when $\xi=0.2$. The form if the wave were propagated in the gas alone is given for reference.

In the above, the mixture, for small values of $\xi$, may be thought of as a fluid containing a uniform distribution of voids or regions of small density. The case where the fluid contains a uniform distribution of regions of high density (with the equivalent of a small $\xi$ ) has recently been studied by Saffman ${ }^{7}$ ) from a stability point of view. In view of the comparatively large second viscosity in the liquid-gas mixture a stability study in this case would also be of interest. The problem with a shock wave in a dusty gas considered by Carrier ${ }^{8}$ ) has analogy with the Campbell and Pitcher work.

Received 28th February, 1963.

\section{REFERENCES}

1) Mallock, A., Proc. Roy. Soc. A84 (1910) 391.

2) Hsieh, D., and M. S. Plesset, Cal. Inst. Tech. Eng. Div. Rep. 85-17, 1960.

3) Campbell, L. J. and A. S. Pitcher, Proc. Roy. Soc. 243 (1958) 534.

4) Symposium, Proc. Roy. Soc. A226 (1954) 1.

5) Taylor, G. I., Proc. Roy. Soc. A2a6 (1954) 34.

6) Davies, R. O., Proc. Roy. Soc. A226 (1954) 39.

7) Saffman, P. G., J. Fluid Mech. 13 (1962) 120.

8) Carrier, G. F., J. Fluid Mech. 4 (1958) 376. 